\title{
Fatigue lifetime prediction methodology of rubber components
}

\author{
C. S. Woo \& W. D. Kim \\ Department of Nano Mechanics Team, \\ Korea Institute of Machinery \& Materials, Korea
}

\begin{abstract}
Interest in the useful lifetime for rubber components increases where the extension of a warranty period is considered. Fatigue analysis and lifetime evaluation are very important in design procedures to assure the safety and reliability of automotive rubber components. The fatigue lifetime prediction methodology of vulcanized natural rubber was proposed by incorporating the finite element analysis and fatigue damage parameter determined from a fatigue test. Nonlinear finite element analyses of the 3-dimensional dumbbell specimen and rubber component were performed using the Ogden hyper-elastic material model determined from the tensile, shear and biaxial tension tests of the natural rubber, and resulted in relationships between displacement and Green-Lagrange strain for both the dumbbell specimen and the rubber component. Fatigue life tests were performed using the 3-dimensional dumbbell specimen at different levels of mean displacement. The fatigue life curve of the natural rubber represented by the maximum Green-Lagrange strain was determined from the finite element analysis and displacement-controlled fatigue test of the dumbbell specimen. Predicted fatigue lives of the rubber engine mount agreed fairly well with the experimental fatigue lives.
\end{abstract}

Keywords: rubber component, fatigue test, fatigue life, finite element analysis, maximum Green-Lagrange strain, life prediction.

\section{Introduction}

Rubber's ability to withstand very large strains without permanent deformation or fracture makes it ideal for many applications. Applications include vibration isolators, seals and structural bearings, to name a few. These applications impose 
large static and time-varying strains over a long time. Long-time durability is therefore a critical issue. While many factors contribute to long-time durability, mechanical fatigue and the nucleation and growth of cracks in the rubber are often the primary consideration [1]. To address the issue effectively and economically, engineers need to model and design for mechanical fatigue early in the product development process. Most rubber components are subjected to static and dynamic loadings in service. Preventing failures during operation is one of the most critical issues in rubber component design [2]. Therefore, fatigue analysis and lifetime evaluation are very important in design procedure to assure the safety and reliability of mechanical rubber components. In order to get an excellent result for fatigue lifetime and have a short time test cycle, the development of new methods for fatigue testing is needed. As a result, we have studied in order to obtain a new fatigue test, which is able to predict the fatigue life for rubber components by the use of correlations with actual vehicle parts by the conventional fatigue test, at the stage of the material development test. This paper shows the fatigue test by a newly designed test piece, which is supposed to simulate actual parts strain by the finite element analysis method, and also show the relationship between the test piece fatigue life and actual parts, using FEM analyzed strain as a parameter. In this study, by using the parameter of maximum Green-Lagrange strains appearing at the locations, we can prove the relations between fatigue life and maximum Green-Lagrange strain, and the correlations between the test piece test and the bench test of actual rubber components. In order to predict the fatigue life of rubber components at the design stage, the simple procedure of life prediction is suggested in Fig.1.

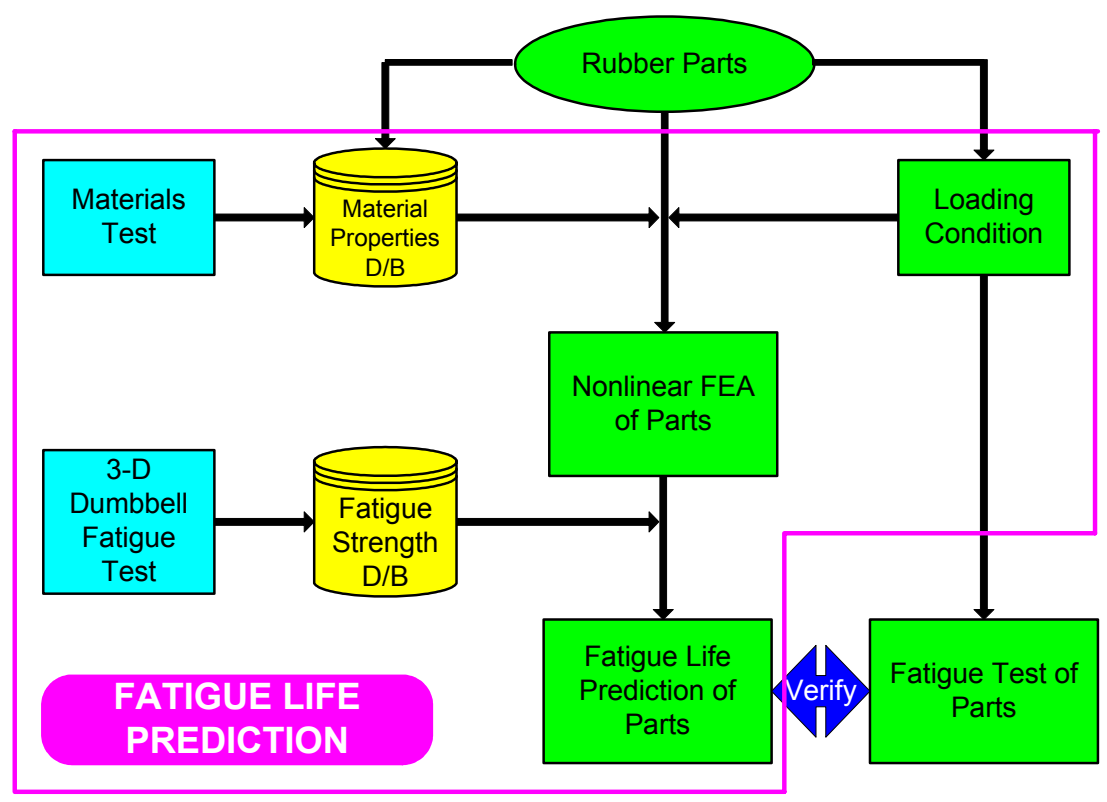

Figure 1: $\quad$ Procedure of fatigue life prediction system for rubber components. 


\section{Experiments}

\subsection{Design of test piece geometry}

The test piece has the basic shape of the 3-dimensional dumbbell specimen with a metal fitting cure bonded to each end. The geometry of the central part of the cylinder was designed to meet the following criteria in relation to fatigue test data for rubber components and strain distribution profile. The test piece should be capable of compression and tensile deformation without developing slackness under cyclic deformation. It should have a smooth strain distribution and the position at which maximum tensile strain develops should be the same for any deformation. The 3D dumbbell specimen has an elliptical cross-section and parting lines are located on the minor axis of the specimen to avoid undesirable failure at the surface discontinuities [3]. The basic geometry of the test piece for materials fatigue testing is shown in Fig. 2. The following FEM analysed strain in terms of maximum tensile and compression strain using ABAQUS software to select the geometry of the curvature and central portion of the test piece. Strain was calculated using the Green-Lagrange strain. Fig. 3 shows the strain distribution according to FEM analysis from the 3D dumbbell specimen in compression and tension. Maximum Green-Lagrange strain was found to develop at a constant position in the surface at the centre of the rubber part of the test piece in both compression and tension.
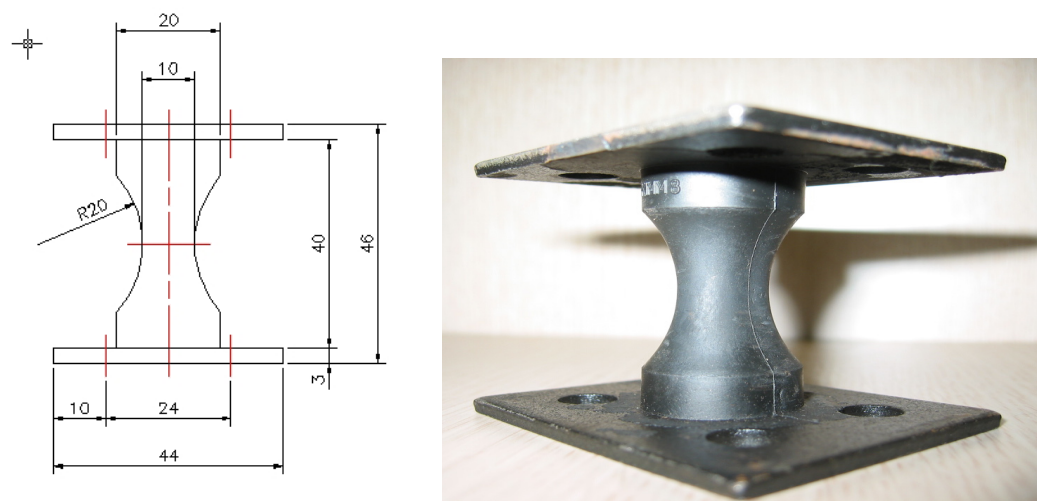

Figure 2: $\quad$ Basic scheme of the 3D dumbbell specimen.

\subsection{Fatigue test of 3D dumbbell specimen}

In order to evaluate a fatigue damage parameter of the natural rubber material and to determine the experimental fatigue life, fatigue tests of the $3 \mathrm{D}$ dumbbell specimen were performed using the fatigue testing system. The material used in this study is a carbon-filled vulcanized natural rubber, which has the hardness of the International Rubber Hardness Degree 50, 55, 60, 65 (NR50, NR55, NR60, NR65). Compound recipes, including applied cure conditions, are summarized in Table 1. Fatigue tests were conducted in an ambient temperature under the 

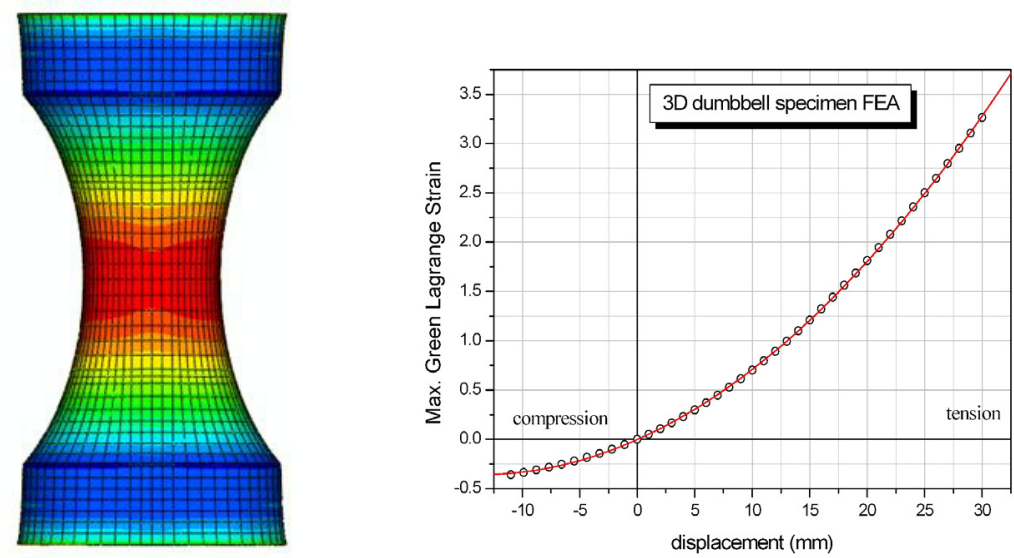

Figure 3: Calculated strain-distribution of the 3D dumbbell specimen.

Table 1: $\quad$ Compound recipes investigated for natural rubber.

\begin{tabular}{|c|c|c|c|c|}
\hline Ingredient & NR50 & NR55 & NR60 & NR65 \\
\hline SMRCV 60 & 100 & 100 & 100 & 100 \\
\hline C/B FEF & 22 & 27 & 40 & 40 \\
\hline C/B SRF & 15 & 18 & 20 & 32 \\
\hline S/A & 1 & 1 & 1 & 1 \\
\hline ZnO & 5 & 5 & 5 & 5 \\
\hline
\end{tabular}

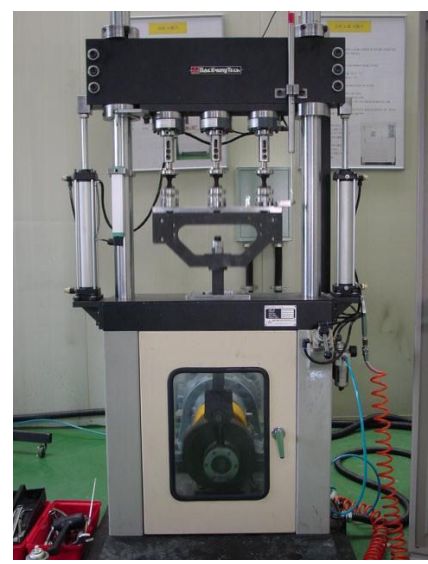

(a) Fatigue testing system

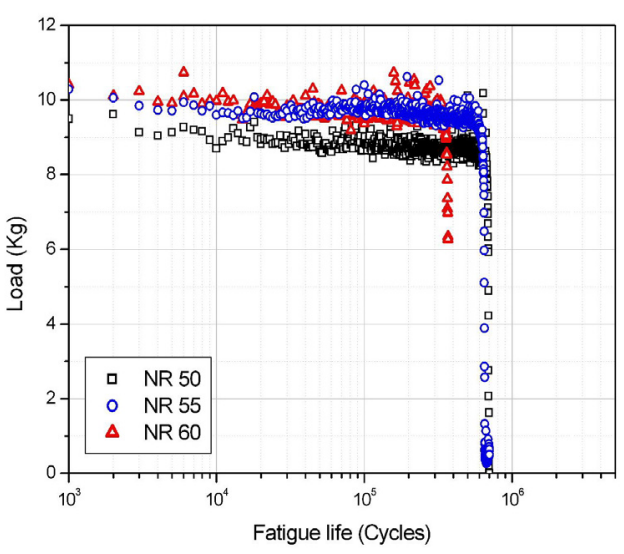

(b) Definition of fatigue failure

Figure 4: $\quad$ Fatigue test of the 3D dumbbell specimen.

stroke-controlled condition with a sine waveform of $5 \mathrm{~Hz}$ and the mean displacement of $0,3,5,8,10 \mathrm{~mm}$ at the displacement range of $-11 \sim 21 \mathrm{~mm}$. The fatigue failure was defined as a number of cycles at which the maximum load 
dropped by 20 percent. As the cycles increased in the initial phase, the maximum load decreased little by little. When the crack grew over the critical size, the maximum load decreased suddenly and the final failure was reached. Fig. 4 shows the fatigue testing system and the fatigue life curve. Fig. 5 shows the relationship between the displacement amplitude and the fatigue life of 3D dumbbell specimens. The fatigue life at the same displacement amplitude decreases as the mean displacement and hardness increases.

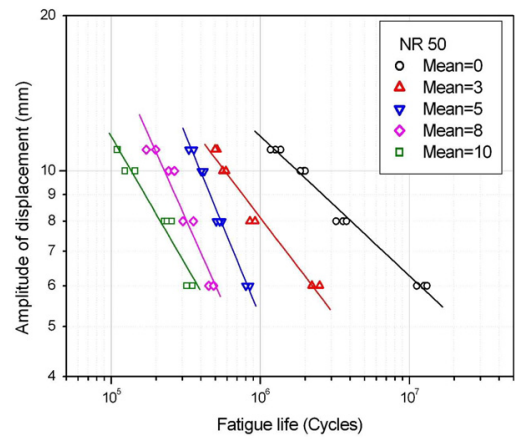

(a) NR 50

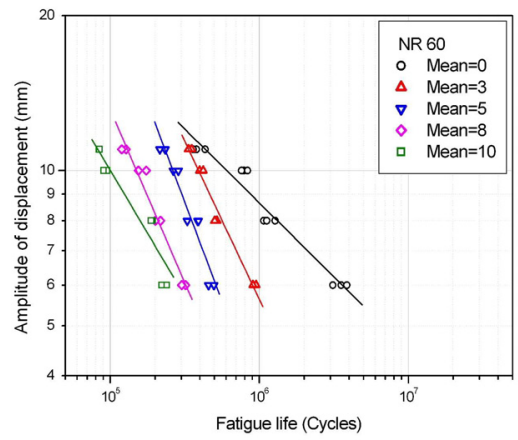

(c) NR 60

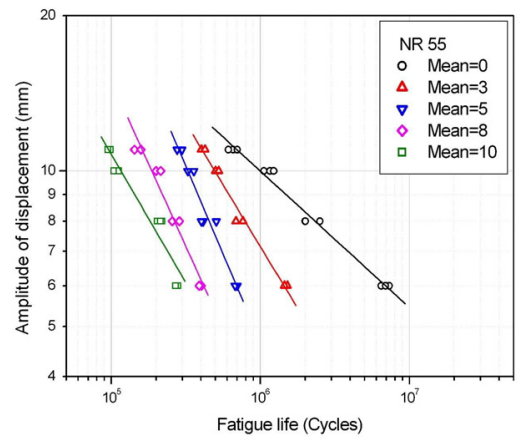

(b) NR 55

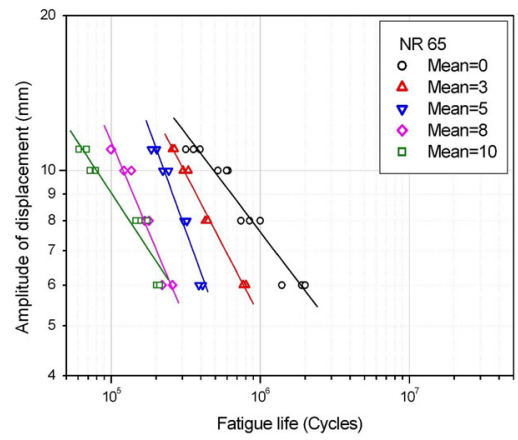

(d) NR 65

Figure 5: $\quad$ Fatigue life of the 3D dumbbell specimen.

\section{Fatigue lifetime prediction and evaluation}

\subsection{Fatigue damage parameter}

The fatigue process begins with the accumulation of damage at a localized region due to alternating load and displacement, leading to crack nucleation, growth, and final fracture $[4,5]$. The crack nucleation life of the component may be defined as the number of cycles required for the appearance of a macro-crack. Therefore, the crack nucleation life of the component can be related to the life of a smooth specimen that is cycled to the same stresses or strains as the material at 
the critical region of the component. In this study, the fatigue damage of the natural rubber was evaluated from smooth dumbbell specimens. Fig. 6 shows the relationship between the maximum tension displacement and fatigue life of the 3D dumbbell specimen. The fatigue life decreases as the maximum tension displacement increases. It is possible to express the fatigue life fairly well with the maximum tension displacement. The relationship between the applied displacement and the corresponding Green-Lagrange strain of the 3D dumbbell specimen are obtained from the finite element analysis and shown in Fig. 3. The fatigue life curve of natural rubber expressed by the Green-Lagrange strain as a damage parameter can be generated from the displacement versus Green-Lagrange strain curve of the 3D dumbbell specimen. Fig. 6 shows the maximum Green-Lagrange strain versus cycles to failure of natural rubber.

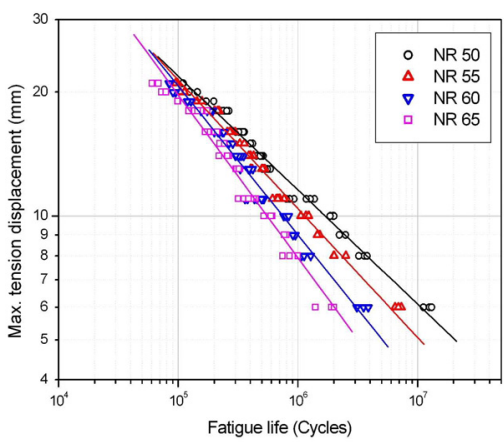

(a) Maximum displacement

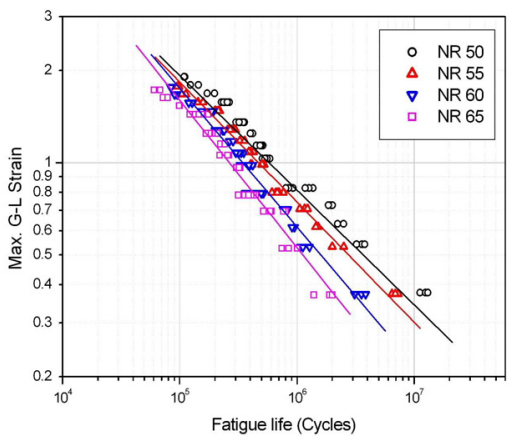

(b) Maximum G-L strain

Figure 6: Maximum displacement and G-L strain versus cycles to failure.

\subsection{Fatigue lifetime prediction}

Fatigue lives of the 3D dumbbell specimen represented by the maximum GreenLagrange strain parameter $\left(\varepsilon_{\mathrm{G}-\mathrm{L}}\right)$ are shown in Fig. $6(\mathrm{~b})$, where the GreenLagrange strain for each dumbbell specimen is calculated from the displacement versus Green-Lagrange strain curve in Fig. 6(a). It can be seen from Fig. 6(b) that the fatigue lives with a different hardness can be effectively represented by the following function using the maximum Green-Lagrange strain, thus taking into account the mean displacement and amplitude. By using the fatigue test and finite element analysis, the normalized maximum strain is defined as dividing by elongation at break $\left(\varepsilon_{\mathrm{EB}}\right)$ for the maximum Green-Lagrange strain $\left(\varepsilon_{\mathrm{G}-\mathrm{L}}\right)$ for each hardness. Fig. 7 shows the relation of normalized maximum strain and fatigue life. It was observed that the maximum G-L strain was a good parameter to account for the hardness, mean displacement and amplitude effects. Fatigue lives of the 3D dumbbell specimen represented by the maximum G-L strain and elongation at break are shown in equation (1). The fatigue lives are effectively represented by a single function using the maximum G-L strain and elongation at break for each natural rubber material. 


$$
N_{f}=1,096 \cdot\left[\varepsilon_{G-L} / \varepsilon_{E B}\right]^{-2.22}
$$

\subsection{Fatigue lifetime evaluation of rubber components}

The methodology was applied to the fatigue life estimation of the rubber engine mount. Finite element analysis was performed to investigate the deformation behaviour of rubber components. Material constants representing the Ogden strain energy potential of order 3 were used for defining a constitutive relation of the natural rubber. Fig. 8 shows the Green-Lagrange strain distribution of a rubber engine mount under a tensile displacement. The maximum GreenLagrange strain at the critical location was used for evaluating the fatigue damage parameter of the rubber engine mount. Static and fatigue tests were performed using the servo-hydraulic fatigue testing system shown in Fig. 9.

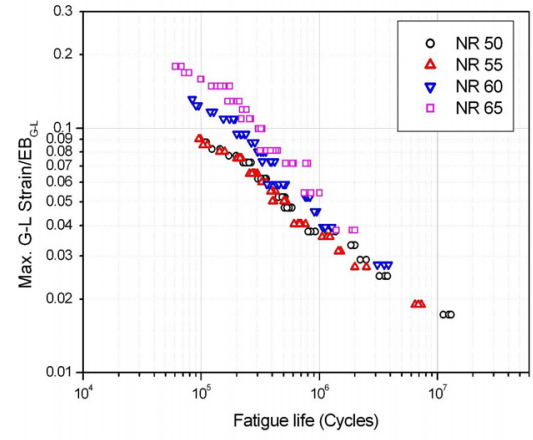

(a) Max. G-L strain versus fatigue life

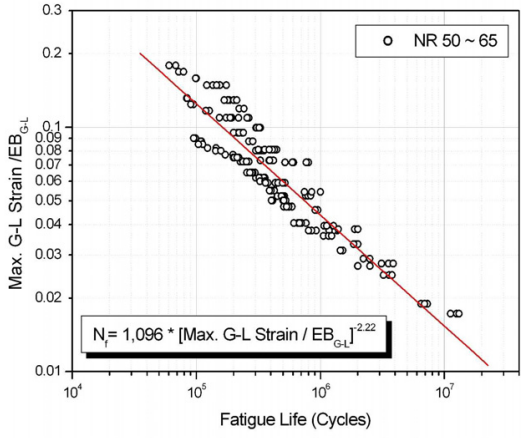

(b) Fatigue life prediction curve

Figure 7: $\quad$ Fatigue lifetime prediction curve of natural rubber.

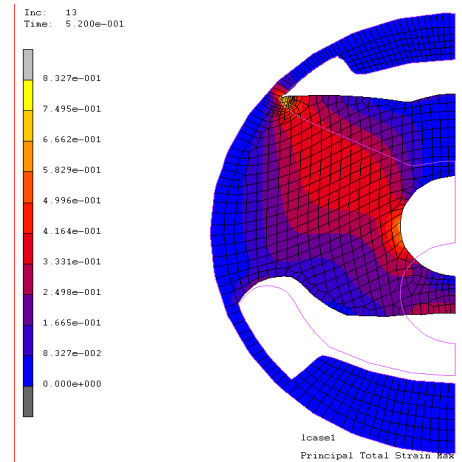

(a) Finite element analysis

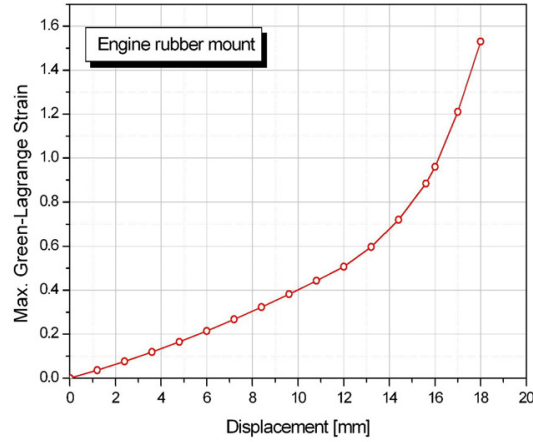

(b) Max. G-L strain versus displacement

Figure 8: Green-Lagrange strain distribution of the engine rubber mount. 


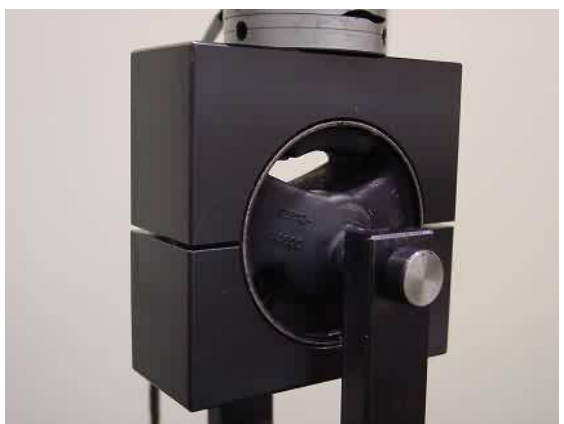

(a) Static test

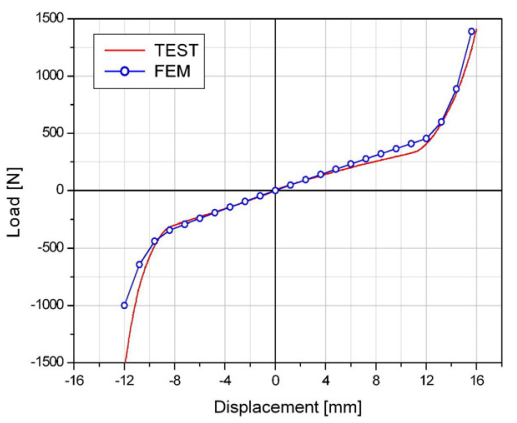

(b) Correlation between test and FEM

Figure 9: Static test and correlation between test and FEM.

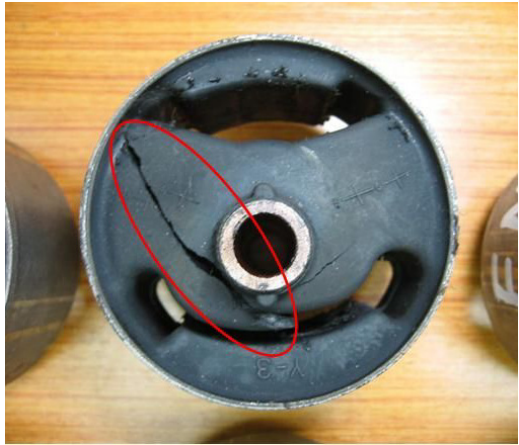

(a) Fatigue failure of engine mount

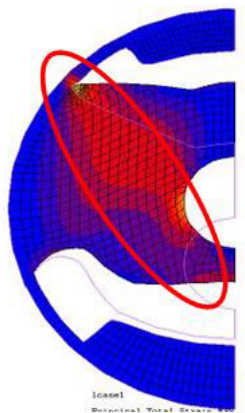

(b) Distribution of G-L strain

Figure 10: Fatigue test and failure of the engine rubber mount.

The load-displacement curve is very similar to the correlation between the test and the FEM. The maximum Green-Lagrange strain occurred at the location as indicated in Fig. 10, and fatigue cracking at the critical location was observed during the fatigue test of the rubber engine mount. Correlation between the fatigue test life and the predicted life of the rubber engine mount using the normalized maximum strain is shown in Fig. 11. The predicted fatigue lives of the rubber engine mount agreed fairly well with the experimental fatigue lives.

\section{Conclusion}

Fatigue lifetime prediction methodology of the rubber components was proposed by incorporating the finite element analysis and fatigue damage parameter. The fatigue life of rubber material was effectively represented by the maximum Green-Lagrange strain. Predicted fatigue lives of the rubber component were in fairly good agreement with the experimental lives. Therefore, the fatigue life estimation procedure employed in this study could be used approximately for the fatigue design of rubber components at the early design stage. 


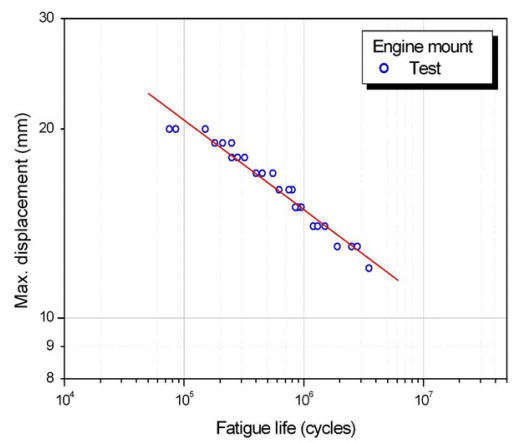

(a) Max. displacement - fatigue life

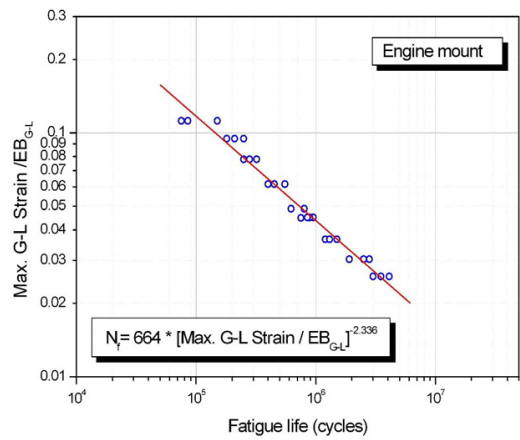

(c) Fatigue life of engine mount

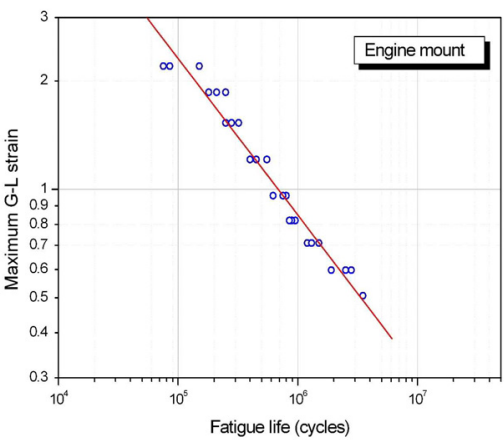

(b) Max. G-L strain - fatigue life

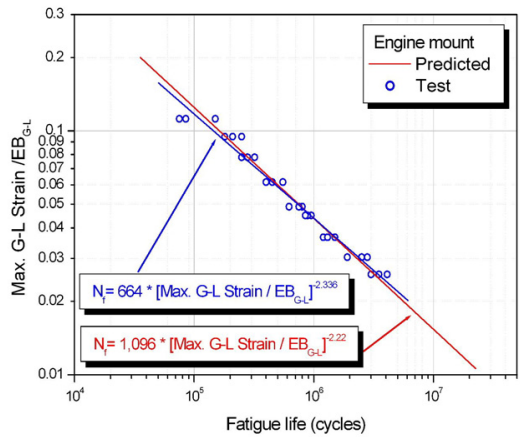

(d) Comparison of predicted and test

Figure 11: Evaluation of fatigue life for the rubber engine mount.

\section{Acknowledgement}

This research has been supported by the Development of Materials and Component program of the Ministry of Commerce Industry \& Energy, Korea.

\section{References}

[1] Gent, A.N, Strength of Elastomer, Science and Technology of Rubber, Mark JE, $2^{\text {nd }}$ ed., Academic Press, Chapter No. 10, pp. 471-512, 1994.

[2] Lake, G.J, Fatigue and Fracture of Elastomers, Rubber Chem. \& Technology, vol. 68, pp. 435-460, 1995.

[3] Yamaguchi, H. \& Nakagawa M., Fatigue Test Technique for Rubber materials of Vibration Insulator, International polymer Science and Technology, vol. 20, p 64-69, 1993.

[4] Oh, H.L, The fatigue life model of rubber bushing, Rubber Chem. \& Technology, vol. 53, pp. 1226-1238, 1980.

[5] Alshuth, T. \& Abraham F., Parameter Dependence and Prediction of Fatigue life of Elastomers products, Rubber Chem. \& Technology, vol. 75 pp. 635642, 2002. 